\title{
Smart Amplify and Forward Relaying in Time and Frequency Diversity Systems
}

\begin{abstract}
Nelly M. Hussein
Abstract: Long distances transmission in mobile communication is considered serious problem especially in $3^{\text {rd }}$ and $4^{\text {th }}$ generations for mobile systems. In order to keep service efficiency in long distance similar to small distance case, two possible solutions are proposed; first is by applying efficient amplification and equalization stages at receiving end. Second solution is by applying relaying network between source and destination. In this paper two relaying techniques were introduced; amplify and forward (AF) relaying and smart AF relaying. Both mention relaying techniques have been simulated using MATLAB program in case of MIMO CDMA and OFDM systems.
\end{abstract}

Keywords: MATLAB, MIMO, CDMA, OFDM, amplify and forward (AF), Wireless Sensor Networks (WSN).

\section{INTRODUCTION}

Recent advances in the development of Wireless Sensor Networks (WSNs) [13] reveal a new paradigm for monitoring infrastructure health [14], [15] and environmental conditions [16] owing to the availability of low powered millimeter-scale CPUs, highly integrated wireless transceiver circuits and various miniature sensors. These provide end users with benefits such as inexpensive installation and maintenance costs, and easy network scalability compared with a wired network. Therefore, the deployment process can be rapid and flexible. Large civil engineering infrastructure such as bridges, high ways, and tunnels are expected to last for decades or even centuries. However, most of them suffer from significant levels of deterioration [17].

A group of researchers from the University of Cambridge are applying WSN technology for long-term continuous monitoring of underground railway tunnels so that efficient risk management system can be established. Therefore, early warning regarding any potential damage of the infrastructure can be promptly reported. Wirelessly gathered data will be analyzed remotely for prediction of catastrophic collapses or tunnel movement.

However, a real deployment of operational WSNs is a challenging task and aspects such as routing protocols, fault tolerance, scalability, data integrity and network lifetime need to be addressed. Fundamentally, sensing coverage and radio connectivity among the wireless sensor nodes in the field of interests are the primary concerns in all applications [18], [19], [20], [21] etc. Underground tunnels are extremely Radio Frequency (RF) hostile owing to their geometry and the roughness of the tunnel walls. The underground wireless channel is one of the main factors that make realizing WSNs a challenge [16] in the tunnel environment. Therefore, an accurate and appropriate radio propagation model for the prediction of the link connectivity is paramount in the planning and deployment of WSNs if acceptable Quality of Service (QoS) is to be achieved. A particular application plays an important role in determining network topology, for example, a multi-hop clustered network is effective for the deployment of WSNs in long and usually empty tunnels. Direct transmission from data source to sinks is usually not practical because sinks are generally far away from the data sources and the Sensor Nodes (SNs) have a limited communication range. Therefore, a multi-hop network is a good choice for data routing, and the clustering topology is appropriate to achieve network scalability.

Careful node placement is important for successful deployment of WSNs while meeting QoS requirements. The network architecture under consideration consists of SNs, Relay Nodes (RNs) and a Base Station (BS). SNs located in specified predetermined sensing locations send the gathered physical information to their local cluster head, i.e., a RN, which in turn forwards the data to the BS either directly or via multi-hop routes. Multi-hop wireless networking traditionally has been studied in the context of ad hoc and peer-to-peer networks. The application of multi-hop networking in wide-area cellular systems has the following benefits. While conventional cellular networks are assumed to have cells of diameter $2-5 \mathrm{~km}$, a relay will only be expected to cover a region of diameter 200-500 m. This means that the transmit power requirements for such a relay are significantly reduced compared to those for a base station (BS). This in turn permits economical design of the amplifier used in the relay. Furthermore, the mast on which the relay is placed does not need to be as high as for a BS, reducing operating expenses such as tower leasing and maintenance costs for the service provider. [1]

\section{MOBILE RELAY OVERVIEW}

Relays are considered as enhancements for the traditional cellular architecture. Relay Stations can intelligently relay data between the BS and mobile stations wirelessly. The communication between the BS and RSs takes place in a similar way as the communication between BS and MSs using point-to-multipoint (PMP) connectivity. In other words, the $\mathrm{RS}(\mathrm{s})$ maintains a wireless backhaul connectivity with the BS and hence the network. At the other end, the RS can establish PMP connectivity with the MS. Therefore, the RS can provide both uplink and downlink connectivity for the MS. The BS-RS links and RS-RS links are usually referred to as relay links whereas 
the BS-MS links and RS-MS links are referred to as access links.

Relay Station proves beneficial in several ways in the cellular network. First, RS provides increased capacity by increasing the frequency reuse. In other words, capacity increase can be realized when both BS and RS in a given area communicate with different MSs using the same frequency resources. Second, it can provide improved coverage with less deployment costs as against fem to BS from the fact that relay uses wireless backhaul link with the network.

The key features of relays such as low equipment cost and flexible deployment options has resulted in strong interest from the industry as well as academia to focus on relay mobility. More intriguing is the fact that mobile relays can potentially be leveraged to achieve new levels of seamless user mobility experience. We highlight three important use cases for leveraging mobile relays to realize some key International Mobile Telecommunications (IMT-Advanced) requirements. These use case are discussed as follows:

1) Group Mobility: First, we highlight group mobility where an improved handover mechanism enables seamless mobility of a group of users. Group mobility makes sense for concurrent handovers to be performed for a group of users in high speed vehicles such as trains, buses. The idea is to have a mobile relay station in the high speed vehicle serving the MSs within. Mobility results in a handover of the relay station to a neighboring BS. At the same time, from the perspective of the MSs, the point of attachment, i.e., RS remains the same. Hence, the PMP connectivity for the MSs is preserved while the RS handover procedure is performed transparent to MSs. Group mobility can significantly reduce the overheads on the radio interface and network thus minimizing latency for all users. In addition, this improved handover mechanism can significantly reduce the $\mathrm{HO}$ interruption time which is one of the key requirements for IMTAdvanced systems.

2) Reliability: The mobile relay concept can also be applied to improve relay network reliability. Since RS is attached to the core network with relay link as wireless backhaul, its reliability is less than typical wired backhaul. Reliability may be a major issue for low-cost small-cell RSs. Ideally, radio link failure prevention and recovery at RS should be handled transparent to the attached MSs. With the mobile relay framework, an RS that is about to experience or just experiences radio link failure on relay link is capable of re-establishing backhaul connection with another suitable neighbor BS using a handover procedure, and this just appears as a scheduling glitch for all MSs associated with RS. Therefore, such self-healing wireless backhaul operation significantly improves network reliability without incurring any special handling at MS.

3) Wireless Backhaul Load Balancing: Similar to the use case of enhancing relay network reliability, the network may even more aggressively switch point of attachment for the RS based on operation status, such as loading of different BS and the associated network gateway. The network may initiate handover for RS to a more suitable BS to ensure load balancing within radio access network as well as core network. Again with mobile relay framework, the network can dynamically perform such operation without impacting the connectivity of MSs associated with the RS. This provides a very attractive feature for network operators. [3]

\section{MULTI-HOP NETWOK ARCHTICTURE}

Direct transmission from data source to sinks is usually not practical because sinks are generally far away from the data sources and the Sensor Nodes (SNs) have a limited communication range. Therefore, a multi-hop network is a good choice for data routing, and the clustering topology is appropriate to achieve network scalability. Careful node placement is important for successful deployment of Wireless Sensor

Networks (WSNs) while meeting QoS requirements. The network architecture under consideration consists of SNs, Relay Nodes (RNs) and a Base Station (BS). SNs located in specified predetermined sensing locations send the gathered physical information to their local cluster head, i.e., a RN, which in turn forwards the data to the BS either directly or via multi hop routes. [4]

\section{WIRELESS RELAY NETWORK MODEL}

Consider a wireless network with NR +2 nodes which are placed randomly and independently according to some distribution. There is one transmitting node and one receiving node. All the other NR nodes work as relays. This is a practical model for many sensor networks. The transmitter has $\mathrm{M}$ transmitting antennas, the receiver has $\mathrm{N}$ receiving antennas, and the $i^{\text {th }}$ relay node has $\mathrm{NR}_{\mathrm{i}}$ antennas, which can be used for both transmission and reception. Since the transmitted and received signals at different antennas of the same relay can be processed and designed independently, this network can be transformed to a wireless relay network with $\mathrm{R}_{\mathrm{i}}$ single-antenna relay nodes. Therefore, without loss of generality, in our paper, we always assume that every relay node has a single antenna. Therefore, the network can be depicted by Figure 1.

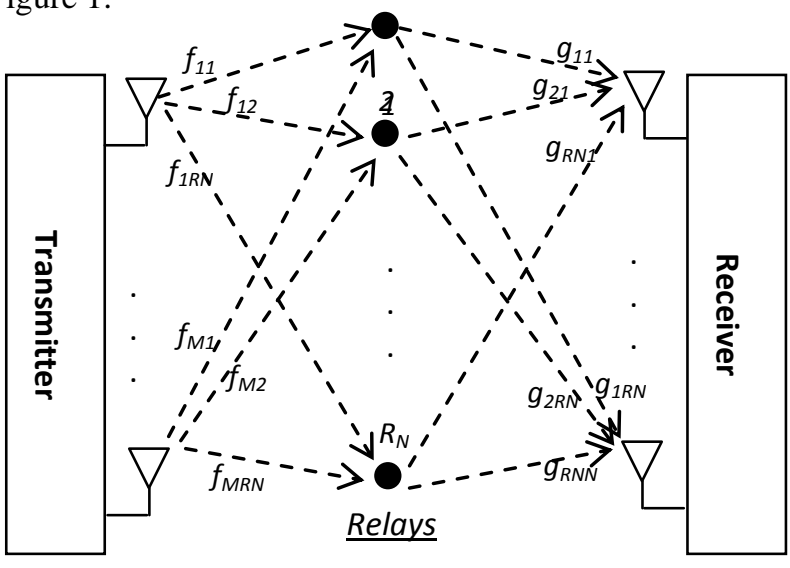

Fig.1 "Wireless relay network with multiple- antenna nodes" 
Denote the channels from the $\mathrm{M}$ antennas of the transmitter to the $i^{\text {th }}$ relay as $\mathrm{f}_{1 \mathrm{i}}, \ldots, \mathrm{f}_{\mathrm{Mi}}$, and the channels from the $i^{\text {th }}$ relay to the $\mathrm{N}$ antennas at the receiver as $\mathrm{g}_{\mathrm{il}}, \ldots, \mathrm{g}_{\mathrm{iN}}$. Here, we only consider the fading effect by assuming that $\mathrm{f}_{\text {mi }}$ and $\mathrm{g}_{\text {in }}$ are independent flat Rayleigh fading complex coefficients. This is a common assumption for urban areas when there is no line-of-sight or when the distances between relay nodes and the transmitter/receiver are about the same. We make the practical assumption that the channels $f_{m i}$ and $g_{\text {in }}$ are not known at the $i^{\text {th }}$ relays. Therefore, transmission of training sequence at the beginning of every signaling interval is necessary for channel estimation at the receiving end. Assume that the transmitter wants to send the signal $\underline{S}=\left[\begin{array}{lll}S_{1} & \ldots & S_{M}\end{array}\right]$ to the receiver. Here, $\mathbf{S}_{\mathrm{m}}$ is a T-dimensional vector and is the signal sent by the $\mathrm{m}^{\text {th }}$ transmit antenna. $\mathrm{T}$ is the coherence interval, that is, the time duration among which the channels $f_{m i}$ and $g_{\text {in }}$ keep constant. Therefore $\underline{S}$ is a T $\times \mathrm{M}$ matrix. As in multipleantenna systems [5], we always assume that $\mathrm{T} \geq \mathrm{M}$. $\underline{S}$ can be either the uncoded information bits or an element from any coded signal set.

\section{RELAYING PROTOCOLS}

Relaying process takes place into two phases. In the first or broadcast phase, the source $\mathrm{S}$ alone broadcasts to the relays and the destination $\mathrm{D}$. In the second cooperation phase, the relays and possibly the source as well, communicate with the destination. A protocol is said to be non-orthogonal or orthogonal depending on whether or not the source continues to transmit in the cooperation phase. The protocol is said to be a decode-and-forward (DF) or an amplify-and-forward (AF) protocol depending upon whether the relays are required to decode the received message or not.

Within the class of DF protocols, one distinguishes between fixed decode-and-forward (FDF) and selection decode- and forward (SDF) protocols. In a SDF protocol, a relay participates in the cooperation phase only if its measurements of the corresponding source-relay channelfading coefficient $\mathrm{g}_{\mathrm{i}}$ reveal the particular source-channel link to lie outside the outage region. In FDF, a relay always decodes. In this paper we will focus on $\mathrm{AF}$ protocol and with some modification, new relaying protocol, denoted by smart AF, will be introduced in the last subsection.

Orthogonality Constraint: In contrast to conventional RF repeaters, small-sized terminals are not capable of receiving and transmitting simultaneously at the same frequency due to hardware imperfections (e.g. oscillations). Hence, relays must be assigned orthogonal resources for their transmission and reception paths. For purposes of exposition, we assume that such separation is performed in the time domain; another approach would be to use frequency relaying where the relays receive and transmit in different frequency bands.

While it is of no concern for our theoretic analysis which of these methods is chosen, it is crucial to note the important consequences of assigning orthogonal sub- channels. Consider time-division relaying as an example. In direct transmission, the source transmits $\mathrm{L}$ symbols in a period $\mathrm{T}$ to the destination. For relay channel coefficients that exceed certain threshold, the source must transmit its L symbols which are followed by the relay's transmission of these $\mathrm{K}$ symbols in the second half period. As a consequence, the spectral efficiency of the individual links must at least be doubled compared to that of the direct system. This holds in the same way for frequency relaying: there, available bandwidth instead of time is shared among the two phases. The impact of such a rate increase is often neglected [9], [10], [12].

Channel Model: The frequency flat fading nature of the channel is captured by coefficients $h$ (including both $\left\{\mathrm{f}_{\mathrm{mi}}\right\}$ and $\left\{\mathrm{g}_{\text {in }}\right\}$ ) drawn from a circularly complex Gaussian distribution: $h \sim C N\left(0, \sigma^{2}\right)$, where $\sigma^{2}$ is the variance of the random process. The magnitudes $|h|$ follow a Rayleigh distribution, and the channel powers $|h|^{2}$ are exponentially distributed with parameter $\sigma^{-2}$. This is a widely accepted channel model; see [8], [10], [12].

Operation of Relays: Relays can enhance the system performance by acting in two different ways:

1) Amplifying relays: Relays that simply amplify their received signals essentially act as active reflectors. This provides diversity gains, and can be understood as enhancing the rank of the MIMO channel matrix.

2) Coding relays: Relays can employ orthogonal coding techniques to enhance the diversity order of the system. An example is the distributed Alamouti method proposed in [11], where two relays retransmit in an amplify-andforward manner, and one of the relays additionally conjugates and negates the signal.

3) Smart amplifying relays: which performs both amplification and data recovery which means it has more complicated hardware stages w.r.t. AF relays. Since we considered in this paper AF relaying and smart AF relaying only, let's explain them in brief.

\section{Amplifying Relays}

In phase 1 , the source transmits a signal vector $X \in$ $\mathbb{C}^{m_{T x} X^{1}}$ from its array where $m_{T x}$ is the number of transmitting antennas. The direct propagation to the destination in the first phase is conventionally described by a matrix $H^{(1, D)} \in \mathbb{C}^{m_{T x} \times m_{R x}}$ where $m_{R x}$ is the number of receiving antennas and a noise vector $n^{(1, D)} \in$ $\mathbb{C}^{m_{T x} \times m_{R x}}$ at the destination, so that the destination's received signal becomes:

$$
y^{(1)}=H^{(1, D)} X+n^{(1, D)} \ldots \quad(1)
$$

Similarly, in this phase each of the $N R^{*}$ relays receives:

$$
r_{l}^{(1)}=H_{l}^{(1)} X+n_{l}^{(1, R)},\left(1 \leq l \leq N R^{*}\right) \ldots(2)
$$

Where, $N R^{*}$ is the number of active relays with channel coefficients that exceed certain threshold, whereas, we denote total number of relays by NR

Where $H_{l}^{(1)} \in \mathbb{C}^{m_{T x} \times m_{R x}}$ is the MIMO matrix mapping the transmitted signal $X$ to the receive antennas of relay $l$, and $n_{l}{ }^{(1, R)}$ is the noise vector at the receive antennas of this relay. To obtain a more compact description, we form the compound signal and noise row vectors: 


$$
\begin{array}{cc}
r^{(1)}=\left[r_{1}^{(1) T}, \ldots,\right. & \left.r_{N R^{*}}^{(1) T}\right]^{T} \\
n^{(1, R)}=\left[n_{1}^{(1, R) T}, \ldots,\right. & \left.n_{N R^{*}}^{(1, R) T}\right]^{T}
\end{array}
$$

and a block channel matrix

$$
H^{(1)}=\left[H_{1}^{(1) T}, \ldots, \quad H_{N R^{*}}^{(1) T}\right]^{T}
$$

where $[.]^{T}$ denotes the transpose of a matrix. Now let's express composite vector received by all $N R^{*}$ relays as follows:

$$
r^{(1)}=H^{(1)} X+n^{(1, R)}
$$

In phase 2, the relays retransmit an amplified version $G . r^{(1)}$ of their received signals to the destination; $G \in \mathbb{C}^{N R^{*} m_{r e l} \times N R^{*} m_{r e l}}$ is the diagonal gain matrix that is conditioned so as to obey the power constraints. Where, $m_{r e l}$ is the number of receiving/transmitting antennas at each relay. These signals undergo fading from relay antennas to the destination; we denote this by a block channel matrix $\mathrm{H}^{(2)} \in \mathbb{C}^{\mathrm{m}_{\mathrm{Rx}} \times \mathrm{NR}^{*} \mathrm{~m}_{\text {rel }}}$. The destination receives:

$$
\begin{gathered}
\mathrm{y}^{(2)}=\mathrm{H}^{(2)} \mathrm{Gr}^{(2)}+\mathrm{n}^{(2, \mathrm{D})} \\
=\mathrm{H}^{(2)} \mathrm{GH}^{(1)} \mathrm{X}+\mathrm{H}^{(2)} \mathrm{Gn}^{\left(1, N R^{*}\right)}+\mathrm{n}^{(2, \mathrm{D})} \\
=\mathrm{H}^{(2)} \mathrm{GH}^{(1)} \mathrm{X}+\left[\begin{array}{ll}
\mathrm{H}^{(2)} \mathrm{G} & \mathrm{I}
\end{array}\right]\left[\begin{array}{c}
\mathrm{n}^{\left(1, N R^{*}\right)} \\
\mathrm{n}^{(2, \mathrm{D})}
\end{array}\right] \ldots(4)
\end{gathered}
$$

Note that (1) and (4) describe the signals received at the destination in the two phases. For purposes of further exposition, we combine both phases, obtaining:

$$
\mathrm{y}=\left[\begin{array}{l}
\mathrm{y}^{(1)} \\
\mathrm{y}^{(2)}
\end{array}\right]=\left[\begin{array}{c}
\mathrm{H}^{(1)} \\
\mathrm{H}^{(2)} \mathrm{GH}^{(1)}
\end{array}\right] \mathrm{X}+\left[\begin{array}{ccc}
\mathrm{I} & 0 & \mathrm{O} \\
0 & \mathrm{H}^{(2)} \mathrm{G} & \mathrm{I}
\end{array}\right]\left[\begin{array}{l}
\mathrm{n}^{(1, \mathrm{D})} \\
\mathrm{n}^{(1, \mathrm{R})} \\
\mathrm{n}^{(2, \mathrm{D})}
\end{array}\right] \ldots
$$

This form describes the complete AF protocol over Rayleigh-fading channels for amplifying relays. It remains to discuss the relay power allocation. From (2) we note that the total power received by relay 1 can be computed as the sum of the powers of the faded signals and the noise powers, i.e.,

$$
\mathrm{P}_{\mathrm{l}}=\frac{\mathrm{P}}{\mathrm{m}_{\mathrm{Tx}}} \sum_{\mathrm{i}=1}^{\mathrm{m}_{\mathrm{rel}}} \sum_{\mathrm{k}=1}^{\mathrm{m}_{\mathrm{Tx}}}\left[\mathrm{H}_{\mathrm{l}}^{(1)}\right]_{\mathrm{i}, \mathrm{k}}^{2}+\mathrm{m}_{\mathrm{rel}} \mathrm{N} \ldots
$$

where $\mathrm{P}$ is the transmission power of the source, and $\mathrm{N}$ is the noise power at the receiver branches of the terminals, which we assume to be equal at all terminals. Note that we assume equal transmit powers at the antennas of the source array. A strict and simple interpretation of the energy constraint is to allow a maximum relay power of $\mathrm{P} / \mathrm{NR}^{*}$. For this, the amplification factor of relay 1 becomes:

$$
\beta_{1}=\sqrt{\frac{\mathrm{P} / \mathrm{NR}}{\mathrm{P}_{\mathrm{l}}}}
$$

These values $\beta_{1}$ are obtained from the diagonal gain matrix G.

Smart Amplified Relays: In this case, relays are not only amplifiers and redirecting stage but also data recovery. Two techniques are considered in this paper; the first is a famous example for time diversity which is CDMA technique and the other is an example for frequency diversity which is OFDM technique. According to selected transceiving system, data recovery part is designed in smart AF relay. In spite of hardware additional complexity of such smart relays, system simulation showed better performance using smart relays w.r.t. ordinary amplifying relays when BER is considered as comparison point of view.

\section{MEASURE LINK PARAMETERS}

In order to measure link parameters, we use an intrusive method. The wireless mobile device regularly (every $t$ seconds) broadcasts probe request packets containing a sequence number and the id or MAC address of the wireless mobile device. Each node receives probe request replies with a probe reply message by using unicast transmission and including information such as its id, its position, and any local information regarding the link parameters. We use an intrusive method to get up-to date information regarding link parameters but also to get a consistent and fair view of each link in the surroundings of a mobile device. An additional advantage of using broadcasting of probe request packets is that we can avoid the clock synchronization problem between devices. It is important to notice that the probe packets, request and reply, have a higher access priority than other packets.

Each node computes its new position based on the surrounding link parameters every $\mathrm{k} \times \mathrm{t}$ seconds, where $\mathrm{k}$ is the number of probe packets, to ensure that enough measures are used to get consistent statistics on the link parameter. The wireless mobile device stores the received value and the measurements obtained through the probe reply. A sliding window is used to compute the statistics, and a FIFO policy is used to remove older values of the link parameters. The wireless mobile device compares the values of the link parameter received from the next and the previous hop, Xnext and Xprev. For example, when the considered parameter is the round-trip time $(\mathrm{X}=$ RTT), if Xnext > Xprev, then the wireless device will move toward the next node. The degree of the inequality changes according to the link parameter considered. In this case, we assume that RTT is somehow related to the distance between nodes. In case of multiple flows passing through the same device, the wireless mobile device will move towards the node $\mathrm{i}$ with the maximum RTT.

The link parameter measurements are averaged and used to compute the new position. The mobile device can use measurements from different layers. [2]

\section{SIMULATION RESULTS}

First set of curves shown in figure 2 represents BER performance versus SNR variation considering MIMO CDMA technique. In this figure two relaying techniques were considered; AF relaying and smart AF relaying using two values of processing gain: $M=32$ represented by solid curves and $M=64$ represented by dashed curves. System parameters are set as follows: number of users $\mathrm{K}=10$, total number of relays $\mathrm{NR}=30$, channel coefficients threshold $\mathrm{Th}=1$, number of transmitting antennas $\mathrm{Nt}=2$, and number of receiving antennas $\mathrm{Nr}=2$. As mentioned before, smart amplifying relaying gives 
better performance w.r.t. amplifying relaying. Simulation results showed that smart amplifying relaying can enhance BER performance by about $8 \mathrm{~dB}$ at the same BER level.

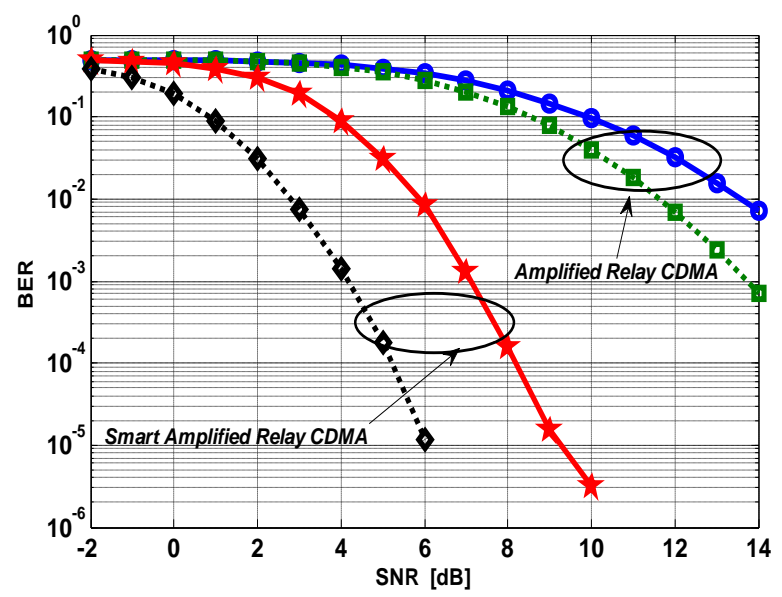

Fig.2: "BER performance vs SNR of CDMA relaying system using amplifying and smart amplifying relays. Solid curve for $\mathrm{PG}=32$ and dashes one for $\mathrm{PG}=64$ "

In the next set of curves, shown in figure 3, relaying techniques were combined with another system which is OFDM transceiving system. Also forward relaying and smart relaying were compared in the presence of MIMO OFDM transceiving system. As mentioned before, multipath Rayleigh fading channel was considered in simulation process. Two channel cases were considered in figure 3; number of channel paths $\mathrm{Lp}=2$, and $\mathrm{Lp}=4$. System parameters in figure 3 are set as follows: IFFT number of points $\mathrm{M}=64$, total number of relays $\mathrm{NR}=$ 45 , channel coefficients threshold $\mathrm{Th}=2.8$, number of transmitting antenna $\mathrm{Nt}=3$, and number of receiving antennas $\mathrm{Nr}=3$.

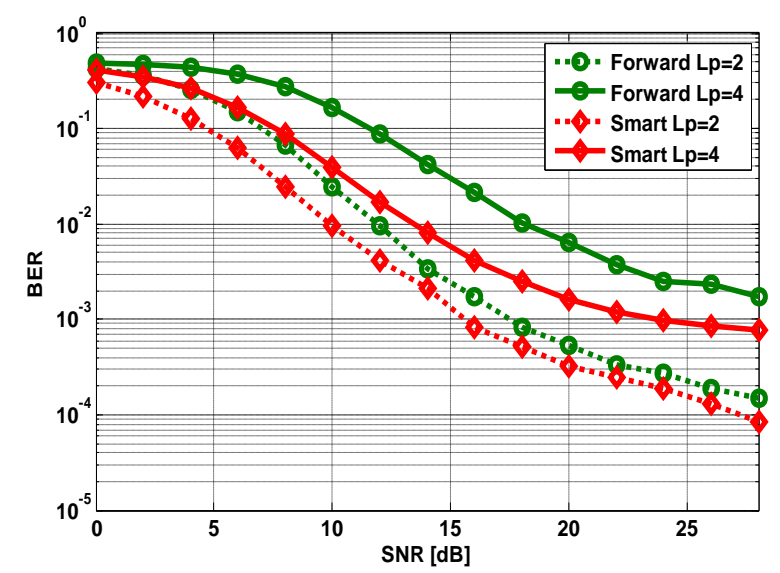

Fig.3: "BER performance vs SNR of OFDM relaying system using amplifying and smart amplifying relays in two cases of fading channels; $\mathrm{Lp}=2$ and $\mathrm{Lp}=4$ "

As mentioned before, not all relays are activated in transferring process but only selected relays with channel coefficients that exceed threshold Th will be activated. Therefore, optimum value of channel coefficients threshold is necessary to be determined in order to obtain satisfactory BER level. In the next set of curves, shown in figure 4, optimum thresholds level required at different MIMO configurations will be displayed in CDMA systems. System parameters are set as follows: $\mathrm{SNR}=15$ $\mathrm{dB}$, number of users $\mathrm{K}=10$, total number of relays $\mathrm{NR}=$ 45 , and BER required is order of $10^{-4}$. The same simulation parameters were repeated in figure 5 as figure 4 but with total number of relays NR $=65$ and considering OFDM system with IFFT number of points $\mathrm{M}=64$.

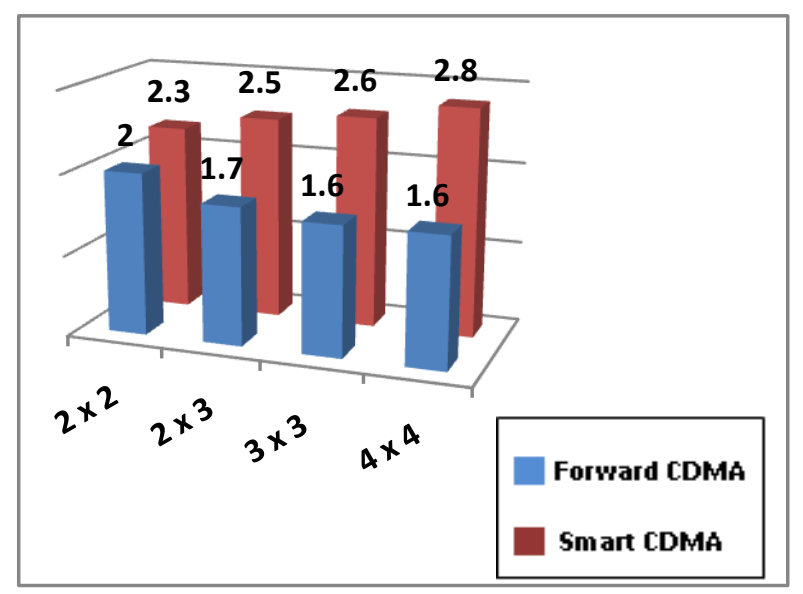

Fig.4: "Optimum channel coefficients threshold in both forward relaying and smart relying in order to reach BER $=$ order of 10-4 at CDMA system"

Last simulation result is displayed in figure 6 in which source- destination transmission delay $T_{d}$ is varying versus total number of relays NR. It's well known that with increment in the number of relays, total displacement executed by transmitted signal will be increased as well. In spite of this fact, system simulation showed that time delay $T_{d}$ varies randomly with total number of relays $\mathrm{NR}$ as illustrated in figure 6 . One explanation for this result is that not all relays are activated at each signaling interval, but only those with channel response amplitude that exceed specific predetermined threshold. And since fading channel is time varying, at every signaling interval the number of

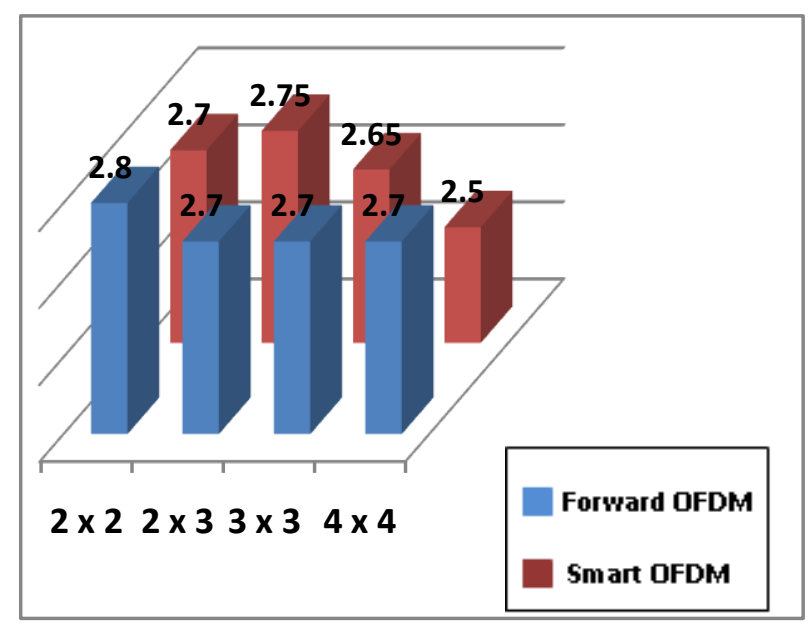

Fig.5: "Optimum channel coefficients threshold in both forward relaying and smart relying in order to reach BER = order of 10-4 at OFDM system" 
active relays will vary with time in random manner. System parameters used in figure 6 , are set as follows: channel threshold $\mathrm{Th}=1.8$, distance between successive relays $L R=50 \mathrm{~m}$, transmission rate $512 \mathrm{~kb} / \mathrm{s}$, and operating frequency $1900 \mathrm{MHz}$.

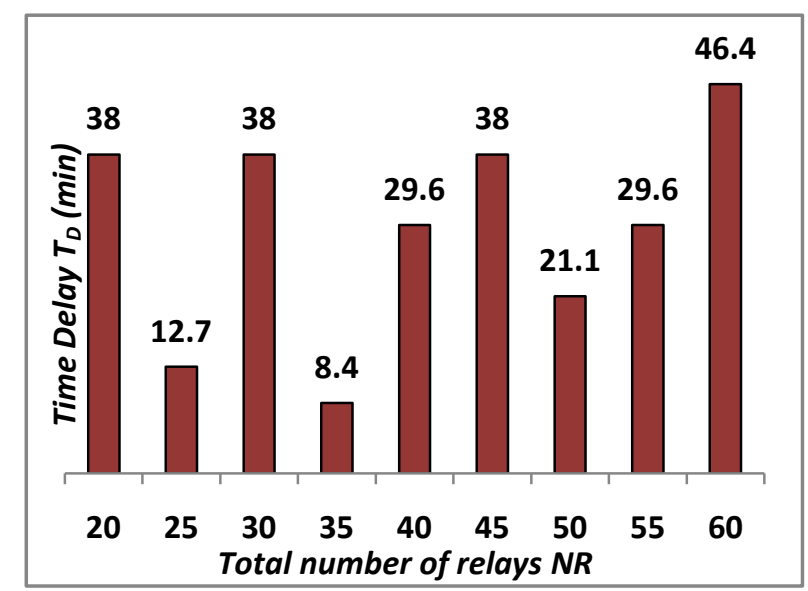

Fig.6: "Total time delays in minutes versus total number of relays"

\section{CONCLUSION}

Relaying techniques have become necessary modification at mobile network for both data and voice efficient reception especially in case of long distances. Among all commonly used relaying mechanisms, amplify and forward (AF) relaying is considered the simplest mechanism. Smart AF relaying includes amplify and forward techniques in addition to data recovery. Simulation results showed that smart amplifying relaying can enhance BER performance by about $8 \mathrm{~dB}$ at the same BER level w.r.t. ordinary AF relaying in both cases of CDMA and OFDM systems.

\section{REFRENCES}

[1]: Ralf Pabst, et al, "Relay-Based Deployment Concepts for Wireless and Mobile Broadband Radio", IEEE Communication Magazine, September 2004.

[2]: Karen Miranda et el, "Adaptive Deployment Scheme for Mobile Relays in Substitution Networks", International Journal of Distributed Sensor Networks Volume 2012, Article ID 128904, 9 pages.

[3]: R. Balakrishnan, et al, "Mobile Relay and Group Mobility for 4G WiMAX Networksq", IEEE WCNC 2011, Intel Corporation.

[4]: Ruoshui Liu, et el , "Relay Node Placement for Wireless Sensor Networks Deployed in Tunnels", 2010 IEEE 6th International Conference on Wireless and Mobile Computing, Networking and Communications.

[5]: T. L. Marzetta and B. M. Hochwald, "Capacity of a Mobile Multiple Antenna Communication Link in Rayleigh Flat Fading," IEEE Trans. Info. Theory, vol. 45, pp. 139-157, Jan. 1999.

[6]: M. Yuksel and E. Erkip,“ Cooperative Wireless Systems: A Diversity-Multiplexing Tradeoff Perspective," submitted to IEEE Trans. Info. Theory, Sep. 2006. Available: http://arxiv.org/pdf/cs.IT/0609122.

[7]: J. N. Laneman, D. N. C. Tse, and G. W. Wornell, "Cooperative Diversity in Wireless Networks: Efficient Protocols and Outage Behavior," IEEE Trans. Inform. Theory, vol. 50, no. 12, pp. 3062 3080, Dec. 2004.

[8]: J. N. Laneman, D. N. C. Tse, and G. W. Wornell, .Cooperative Diversity in Wireless Networks: Ef_cient Protocols and Outage Behavior,. IEEE Trans. Inform. Theory, Oct. 2003, (accepted for publication).
[9]: M. Dohler, F. Said, and H. Aghvami, .Concept of Virtual Antenna Arrays, in Proc. IEEE Globecom 2002, Taipei, Taiwan, 2002.

[10]: M. Dohler, J. Dominguez, and H. Aghvami, .Link Capacity of Virtual Antenna Arrays,. in Proc. IEEE Vehic. Techn. Conf., Sept. 2002.

[11]: P. A. Anghel, G. Leus, and M. Kaveh, Multi-User Space-Time Coding in Cooperative Networks,. in Proc. ICASSP, Hong Kong, Apr. 2003.

[12]: A. Wittneben and B. Rankov, .Impact of Cooperative Relays on the Capacity of Rank De_cient MIMO Channels, in IST Mobile \& Wireless Communications Summit 2003, Aveiro, Portugal, June 2003.

[13]: I. Akyildiz, W. Su, Y. Sankarasubramaniam, and E. Cayirci, "Wireless sensor networks: a survey," Computer networks, vol. 38, no. 4, pp. 393-422, 2002.

[14]: N. Hoult, P. Fidler, I. Wassell, P. Hill, and C. Middleton, "Wireless structural health monitoring at the Humber Bridge UK," Bridge Engineering, vol. 161, no. 4, pp. 189-195, 008.

[15]: C. Park, Q. Xie, P. Chou, and M. Shinozuka, "Duranode: Wireless networked sensor for structural health monitoring," in IEEE Sensors, 2005.

[16]: I. Akyildiz and E. Stuntebeck, "Wireless underground sensor networks: Research challenges," Ad Hoc Networks, vol. 4, no. 6, pp. 669-686, 2006

[17]: F. Stajanoa, N. Houltb, I. Wassella, P. Bennettb, C. Middletonb, and K. Sogab, "Smart Bridges, Smart Tunnels: Transforming Wireless Sensor Networks from Research Prototypes into Robust Engineering Infrastructure," in submission.

[18]: C. Huang and Y. Tseng, "The coverage problem in a wireless sensor network," Mobile Networks and Applications, vol. 10, no. 4, pp. 519-528, 2005.

[19]: S. Meguerdichian, F. Koushanfar, M. Potkonjak, and M. Srivastava, "Coverage problems in wireless ad hoc sensor networks," in IEEE INFOCOM, vol. 3. Citeseer, 2001, pp. 1380-1387.

[20]: S. Shakkottai, R. Srikant, and N. Shroff, "Unreliable sensor grids: Coverage, connectivity and diameter," Ad Hoc Networks, vol. 3, no. 6, pp. 702-716, 2005.

[21]: J. Chang, P. Hsiu, and T. Kuo, "Search-Oriented Deployment Strategies for Wireless Sensor Networks," in Proceedings of the 10th IEEE International Symposium on Object and ComponentOriented Real-Time Distributed Computing, 2007, pp. 164-171.

\section{BIOGRAPHY}

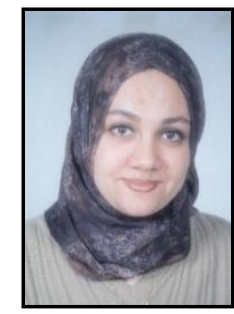

Nelly Muhammad Hussein, Ph.D. from Faculty of Engineering Communication department, Cairo University 2010. Lecturer in Modern Academy for Engineering and Technology, Communication Dept. Published 5 single author papers mentioned as follows:

1. "Applying Channel Equalization Techniques to STBC OFDM - CDMA System in The Presence of Multi-Path Frequency Selective Channel Fading", Vol. 5, No. 4, April 2009.

2. "Performance Enhancement of STBC OFDM- CDMA System Using Channel Coding Techniques over Multipath Fading Channel", Vol. 5 No. 5, May 2009.

3. "LTE System Performance in Frequency Selective Fading Channel with aid of STBC Technology", UNIASCIT, Vol 1 (2), 2011, 54-62.

4. "Wavelet Transform Effect on MIMO-OFDM System Performance", Cyber Journals: Multidisciplinary Journals in Science and Technology, Journal of Selected Areas in Telecommunications (JSAT), September Edition, 2011. 5. "Performance of OFDM- CDMA System using Modified Space- Shift Keying Technique", International Journal of Information and Communication Technology Research, Volume 2 No. 2, February 2012. 